\title{
Calibration 4.0 - Information system for usage of digital calibration certificates
}

\author{
Miguel Marques ${ }^{1, *}$, João A. Sousa ${ }^{2}$, Luís Ribeiro ${ }^{2}$ \\ ${ }^{1}$ INEGI, Universidade do Porto, Rua Dr. Roberto Frias, 400, 4200-465 Porto, Portugal \\ ${ }^{2}$ IPQ, Instituto Português da Qualidade, Rua António Gião, 2, 2829-513 Caparica, Portugal
}

\begin{abstract}
The main goal of this study is to operationalize the emission, exchange and use of digital calibration certificates, through structured information and the specification of a system of information. The purpose of digital calibration certificates, is not only the transformation of a physical format to a digital one, but mainly the application of the real digitalization concept, i.e., the use of digital technologies to change a business model and supply new opportunities with added value. Besides the easiness in the exchange of calibration results, the future development of a system to exchange digital calibration certificates will allow customers to receive and analyze certificates faster, reducing execution times and costs associated to the metrological confirmation process, as well as to avoid human error and their consequences. The application of a system adapted to calibration certificates, and the implementation and testing at a National Measurement Institute as IPQ, based on XML, with examples from different areas will be presented. This study will then result in the creation of national working group in Portugal for the digitalization of calibration certificates to a broader market, with the first trial cases implemented in these two laboratories, INEGI-LAC and IPQ-LNM.
\end{abstract}

\section{Introduction}

In this digital era and Industry 4.0 environment, the results of calibration are still linked to printed documents and manual transcription which difficult the connection to information systems and industrial automation. What is the purpose of having equipment with internet connection if the results of its calibration kept in a paper format? How can a laboratory demonstrate traceability through digital media?

With the purpose of maintaining competitiveness and avoiding laboratories to be stuck to communications based on paper and transcription, it is urgent the adaptation of testing and calibration laboratories to a new approach, adapting to the new reality of Industry 4.0. This will enable the use of technological advances as reported in some applications, at least partially, e.g., EMPIR project Met4FoF [1] and the German VDI initiative [2].

The main goal of this study is to operationalize the production, exchange and use of digital calibration certificates, through structured information and the specification of a system of information. The development of a system adapted to calibration certificates, and the

*Corresponding author: mmarques@inegi.up.pt 
implementation and testing at a National Measurement Institute as IPQ, based on XML, with examples from different areas will be presented. This study will then result in the creation of national working group in Portugal for the digitalization of calibration certificates to a broader market, with the first trial cases implemented in these two laboratories, INEGI-LAC and IPQ-LNM.

\section{Digital Calibration Certificates (DCC)}

The purpose of digital calibration certificates, is not only the transformation of a physical format to a digital one, but mainly the application of the real digitalization concept, i.e., the use of digital technologies to change a business model and supply new opportunities with added value. Besides the easiness in the exchange of calibration results, the future development of a system to exchange digital calibration certificates will allow customers to receive and analyse certificates faster, reducing execution times and costs associated to the metrological confirmation process, as well as to avoid human error and their consequences. The easiness of information analysis and gathering from a sequence of calibrations, allows a deeper analysis of data gathered during the calibration.

One of the main advantages the use of digital calibration certificates lies on the possibility of real-time action on the instrumentation. In the factory of the future environment, there will be a large number of low-cost sensors, and thus it will be necessary to use the data from the calibration certificates to check the validity of the measured data in real or quasireal time. For that, the machine-readable format can be use by smart sensors to ascertain the quality of measured data, by accepting the results of calibration or, in alternative, to impose automatic corrections to its readings to achieve the same goal.

An example concerns the process of metrologically accepting the results from a calibration certificate that depends on the intended use of the particular instrumentation (fit-forpurpose concept) and must be based on a decision rule in accordance with the requirements of the new version of standard ISO/IEC 17025:2017. One common decision rule is based on the comparison between manufacturer specifications (sometimes multiplied by a safety factor) and the sum of error (absolute value) and expanded uncertainty, and that example will be illustrated in this study.

\section{The guidelines proposed by the VDI/VDE document}

\section{1. $X M L$ language}

The code proposed in the VDI/VDE 2623 document [2] is based on XML language, and being specifically intended for a calibration process. The Calibration Data Exchange format (CDE) proposed in the document defines a structure in order to collect data, relevant and required for the management of measuring equipment, regarding the processes of commissioning and transfer calibration information. Such a structure is organised from an order, subdivided into two parts: administrative and technical. The administrative part contains the data of the applicant and the supplier of the calibration services. The technical part contains the information regarding the instrument being studied, verified or calibrated and that relating to the calibration process, including calibration results, environment conditions, information on traceability, uncertainty, coverage probability and the like. 
Due to the large diversity of information to be included in each certificate and inherent to each instrument category, the publication by the supplier of an XSD file type will facilitate the recognition and interpretation of such diversity. The XSD format is an XML Schema file: a text-based file format that defines validation rules for an XML file and explains the $\mathrm{XML}$ form, providing the model for the contents of the digital certificate.

Formats for dates [3], units [4], country codes [5], language [6] and the like, shall comply with relevant international standards as well as the use of the period for decimal separator.

Example of the calibration section of a digital certificate in a XML format, with information regarding calibration result, ambient conditions, traceability, used technical procedure, among others.

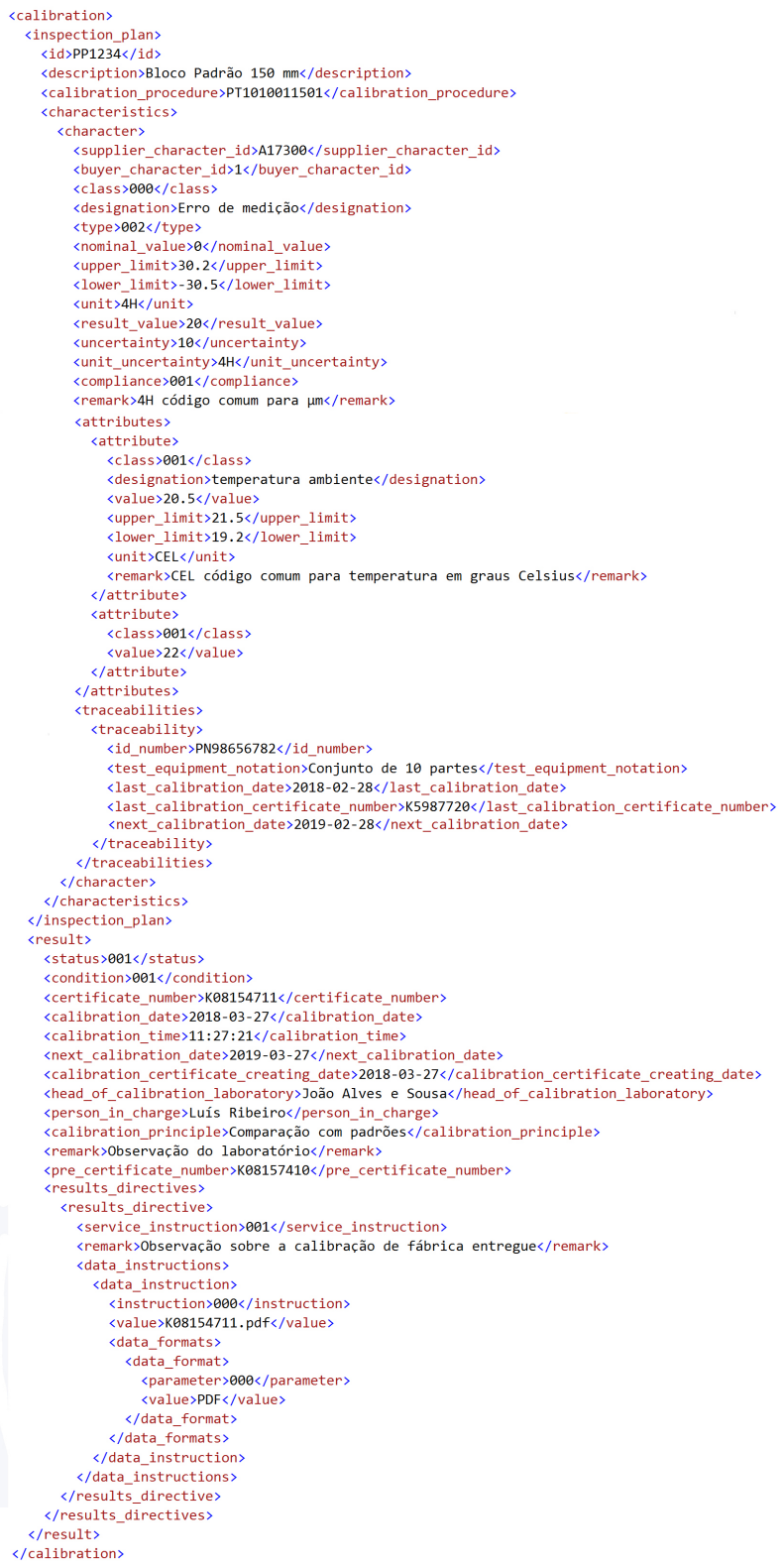




\subsection{Additional considerations}

Applying an information system to an existing system where, despite not fully digitalized, requirements in storage and display of information already exist, some additional and specific requirements are considered [7].

National accreditation bodies specify requirements and specifications for laboratories or certificates. In the Portuguese case, the OGC001 issued by Instituto Português de Acreditação, IPAC, is considered.

Should the accreditation body be signer of a Mutual Recognition Agreement, either with ILAC or IAF, an optional statement attribute must be inserted, example, $<$ MRAstatement $>$.

\subsection{INEGI-LAC case study}

\subsubsection{Anemometer}

INEGI-LAC performs the calibration of air velocity measuring equipment. The case study uses a customer laboratory that frequently calibrates equipment but requires a "just in time" calibration, so that equipment downtime is reduced.

An implementation of the digital certificate "translates" the content of the calibration certificate to file "A.18.012097.xml", attached to the PDF file.

The customer implemented, through the XSD and supporting documents, an automated system that analysis the calibration certificates. Calibration documents are uploaded to the document management system and processed, comparing the results (namely error through 4 to $16 \mathrm{~m} / \mathrm{s}$, correlation coefficient and residual errors of the transfer function) to acceptance criteria. If the result of such analysis is a deviant result, management staff is alerted for further analysis and decision.

Should all be right once the technicians receive the equipment, the calibration certificate has already been processed and the anemometers are ready for deployment. This way, the usage of digital calibration certificates further reduces such downtime by allowing a prompt analysis of the calibration results

\subsection{IPQ-LNM case study}

\subsubsection{Multimeter}

In the case of a multimeter calibration, the complexity arises from the fact that there are several functions, each one with several ranges, that are to be reported, including the determined calibration error, expanded uncertainty, effective number of degrees of freedom, coverage factor [8]. The interpretation or usage of these values varies according the particular application. One foreseen solution in order to facilitate the digital implementation could involve the calculation of the calibration curve associated to each range, allowing a machine interpretation algorithm to take profit of the electronic availability of calibration data.

For example: in an actual common calibration certificate the data is presented in a table format like the example of table 1 . 
Table 1. Data from a multimeter calibration.

\begin{tabular}{ccccc}
\hline $\begin{array}{c}\text { Measurement } \\
\text { function }\end{array}$ & $\begin{array}{c}\text { Measurement } \\
\text { range } / \mathrm{V}\end{array}$ & $\begin{array}{c}\text { Applied value } \\
/ \mathrm{V}\end{array}$ & $\begin{array}{c}\text { Reading error } \\
/ \mathrm{V}\end{array}$ & $\begin{array}{c}\text { Expanded } \\
\text { uncertainty } / \mathrm{V}\end{array}$ \\
\hline DCV & 0.1 & -0.10000000 & $-5.0 \mathrm{E}-07$ & $5.9 \mathrm{E}-08$ \\
DCV & 0.1 & -0.05000000 & $-3.0 \mathrm{E}-07$ & $5.8 \mathrm{E}-08$ \\
DCV & 0.1 & 0.00000000 & $-1.8 \mathrm{E}-07$ & $5.8 \mathrm{E}-08$ \\
DCV & 0.1 & 0.05000000 & $6.2 \mathrm{E}-07$ & $5.8 \mathrm{E}-08$ \\
DCV & 0.1 & 0.10000000 & $7.5 \mathrm{E}-07$ & $5.9 \mathrm{E}-08$ \\
\hline DCV & 1 & -1.0000000 & $-4.7 \mathrm{E}-06$ & $5.9 \mathrm{E}-07$ \\
DCV & 1 & -0.5000000 & $-2.9 \mathrm{E}-06$ & $5.8 \mathrm{E}-07$ \\
DCV & 1 & 0.0000000 & $2.5 \mathrm{E}-07$ & $5.8 \mathrm{E}-07$ \\
DCV & 1 & 0.5000000 & $2.7 \mathrm{E}-06$ & $5.8 \mathrm{E}-07$ \\
DCV & 1 & 1.0000000 & $3.0 \mathrm{E}-06$ & $5.9 \mathrm{E}-07$ \\
\hline DCV & 10 & -10.000000 & $-8.2 \mathrm{E}-06$ & $5.9 \mathrm{E}-06$ \\
DCV & 10 & -5.000000 & $-4.3 \mathrm{E}-06$ & $5.8 \mathrm{E}-06$ \\
DCV & 10 & 0.000000 & $-3.0 \mathrm{E}-07$ & $5.8 \mathrm{E}-06$ \\
DCV & 10 & 5.000000 & $2.3 \mathrm{E}-06$ & $5.8 \mathrm{E}-06$ \\
DCV & 10 & 10.000000 & $5.6 \mathrm{E}-06$ & $5.9 \mathrm{E}-06$ \\
\hline$\ldots$ & $\ldots$ & $\ldots$ & $\ldots$ & $\ldots$ \\
\hline
\end{tabular}

However, the same information could be provided in terms of range gain and range offset that arises from linear, that is, a straight-line calibration function that describe a dependent variable Y (response) as a function of an independent variable X (stimulus), instead of only using the variability in the observed quantity [9]. The straight-line relationship depends on the intercept $\mathrm{A}$ and the slope $\mathrm{B}$ of the line, determined using least squares methods. A and $\mathrm{B}$ are referred to as the parameters of the line where A represents the range offset and $\mathrm{B}$ the range gain, as in table 2 , for the same calibration data.

Table 2. Linear fit parameters from a multimeter calibration.

\begin{tabular}{cc|cc|cc}
\hline \multirow{2}{*}{$\begin{array}{c}\text { Measurement } \\
\text { function }\end{array}$} & \multicolumn{2}{|c|}{ Gain } & \multicolumn{2}{c}{ Offset } \\
\cline { 3 - 6 } & range / V & Error & $\begin{array}{c}\text { Expanded } \\
\text { uncertainty / } \\
V\end{array}$ & Error / V & $\begin{array}{c}\text { Expanded } \\
\text { uncertainty / V }\end{array}$ \\
\hline DCV & 0,1 & $-6,8 \mathrm{E}-06$ & $3,86 \mathrm{E}-07$ & $-7,8 \mathrm{E}-08$ & $2,71 \mathrm{E}-08$ \\
DCV & 1 & $-4,2 \mathrm{E}-06$ & $3,86 \mathrm{E}-07$ & $3,3 \mathrm{E}-07$ & $2,71 \mathrm{E}-07$ \\
DCV & 10 & $-6,8 \mathrm{E}-07$ & $3,86 \mathrm{E}-07$ & $9,8 \mathrm{E}-07$ & $2,71 \mathrm{E}-06$ \\
\hline$\ldots$ & $\ldots$ & $\ldots$ & $\ldots$ & $\ldots$ & $\ldots$ \\
\hline
\end{tabular}

The electronic version of data in an XML format Calibration Certificate could be easily achieved, in order to accommodate the information of one or both options, and the correspondent XSD file format would provide the necessary information for the development and implementation of algorithms, by the owners or users of the calibrated measurement instrument, to interpret the machine-readable code provided.

\subsubsection{Calibration results validation}

The automatic validation of the results presented in a calibration certificate, associated to the process of its metrologically acceptance, that depends on the intended use of the particular instrumentation, is highly facilitated with the use of machine-readable code, allowing the automatic application of any decision rule based on particular specifications. For example: the comparison between manufacturer specifications (sometimes multiplied 
by a safety factor) and the sum of error (absolute value) and expanded uncertainty. Again, the development and implementation of algorithms to interpret the machine-readable code provided in such digital certificates would certainly be an added value for the applicant.

\section{Future work}

All future work requires definition, test, and acceptance through consensus. An ISO/CEN technical committee is the natural place o work for standardization. A standard, adopted by several countries, is required so that other laboratories can implement the same schema and exchange of digital calibration certificates can start.

To the authors VDI/VDE 2623 is the most relevant candidate for a first generation standard, despite national requirements, applicable to calibration certificates and that need consideration. Once a national technical committee defines the XML elements and attributes correspondent to said requirements, a national branch, such as "nationalsection_PT", is included in the XML output.

The development of an open source tool, most likely through a XML-based spreadsheet file format (like XLSX or ODS) would allow laboratories to implement and experiment on their own existing systems.

A Digital Metrological Traceability of results, the unbroken chain of calibrations towards international reference standards requires, the very least, that laboratories are uniquely identified through a digital ID. Hence, standardization should also encompass entities such as "accreditation body", "accreditation certificate" and "laboratory" so that the calibration of a reference equipment used can be shown.

The added value to industry and metrology requires communication and dissemination of the proposed techniques. EMPIR project Met4FoF is an example of such and activities at national level are required.

Future work must also consider the results of field, factory and test laboratories, allowing for a full connection between reference SI units and results.

\section{Acknowledgments}

The EMPIR project "17IND12-MET4FOF" is carried out with funding of the European Union under the EMPIR. The EMPIR is jointly funded by the EMPIR participating countries within EURAMET and the European Union.

\section{References}

1. EMPIR project 17IND12 Met4FoF, "Metrology for the Factory of the Future", https://www.ptb.de/empir2018/met4fof/home/.

2. VDI/VDE 2623, "Format for Data Exchange in Management of Measuring and Testing Equipment”, ICS 03.120.10, 35.240.99 (February 2012). 
3. ISO 8601-1:2019, "Data elements and interchange formats - Information interchange Representation of dates and times".

4. Codes for Units of Measure Used in International Trade, revision 6, (UN/ECE CEFACT trade Facilitation recommendation No. 20), Annex I, http://tfig.unece.org/contents/recommendation-20.htm.

5. ISO 3166-1:2006 "Code for the representation of names of countries and their subdivisions".

6. ISO 639-1:2002-07 "Codes for the representation of names of languages".

7. A. Cardoso, Sistema de informação para troca de certificados de calibração digitais, Master in Services Engineering and Management, FEUP, Universidade do Porto (2018), https://goo.gl/c3oooU.

8. JCGM 100:2008 (GUM 1995 with minor corrections) Evaluation of measurement data - Guide to the expression of uncertainty in measurement. BIPM.

9. ISO/TS 28037, Technical Specification 1, "Determination and use of straight-line calibration functions" (2010). 